\title{
Optimal microstructures. Drag reducing mechanism of riblets
}

\author{
Elfriede Friedmann \\ Interdisciplinary Center for Scientific Computing \\ Ruprecht-Karls-University Heidelberg, Germany \\ friedmann@iwr.uni-heidelberg.de \\ Thomas Richter \\ Department for Aeronautics \&S Astronautics \\ Massachusetts Institute of Technology, USA \\ trichter@MIT.EDU
}

\begin{abstract}
We consider an optimal shape design problem of periodically distributed three-dimensional microstructures on surfaces of swimming bodies in order to reduce their drag. Our model is restricted to the flow in the viscous sublayer of the boundary layer of a turbulent flow. There the flow is described by the incompressible steady-state Navier-Stokes equation with a Couette Flow profile. Because of the geometry, the state equations have oscillating coefficients and are difficult to solve. In the optimization process the equations have to be solved several times so that the costs are very high. We avoid this problem by approximations: the microscopic optimization problem is reduced applying homogenization. The advantage of using this method is that the oscillating equations can be replaced by so-called effective ones with smooth coefficients which are easier to solve, and the oscillating cost function can be replaced by the effective drag which depends only on the so-called Navier matrix. The entries of this matrix can be calculated using an auxiliary boundary layer problem, a Stokes-type equation. The domain of computation is reduced to a small part of the initial domain which also decreases the computation costs. Considering a special geometry (riblets) the resulting so-called macroscopic optimization problem can additionally be reduced to a two-dimensional problem. We analyze the drag reducing mechanism of riblets which are believed to be optimal structures by means of the Navier constants. Finally the macroscopic optimization problem is solved by a sensitivity based method. To validate the homogenized
\end{abstract}


model it is important to perform direct simulations on the total rough geometry for different shapes of microstructures: riblets and fully three-dimensional structures. Because of the complicated domain we have to perform this direct simulations combining error control, adaptive mesh refinement and a fast solution algorithm based on multigrid methods. The discretization of the state equations is done by stabilized finite elements. Comparing the values for the oscillating drag force with the values for the effective drag force which we calculated with the reduced model we get conformity for small scales and we get the information that the reduced model should be used for the optimization process even for higher structures. Then direct simulations have to be performed on the obtained geometry in order to calculate the exact amount of drag reduction, resulting in a decrease up to $10 \%$.

Keywords: Shape optimization, drag minimization, Navier-Stokes equations, Couette flow, boundary layer, homogenization.

\section{References}

[1] D.W. Bechert, M. Bruse, and W. Hage, Experiments with three-dimensional riblets as an idealized model of shark skin, Experiments in Fluids, Springer Verlag, Vol. 28, p. 403-412, 2000.

[2] R. Becker, and R. Rannacher, A feed-back approach to error control in finite elemnt methods: Basic analysis and examples, East-West J. Numer. Math., Vol. 4, p. 237-264, 1996.

[3] R. Becker, and R. Rannacher, An optimal control approach to a posteriori error estimation in finite element methods, Acta Numerica 2001, Cambridge University Press, 2001.

[4] M. Braack and T. Richter, Solutions of 3D Navier-Stokes benchmark problems with adaptive finite elements, Computers \& Fluids, 35(4), p. 372-392, 2006.

[5] E. Friedmann, Riblets in the viscous sublayer. Optimal Shape Design of Microstructures, PhD thesis Ruprecht - Karls - University Heidelberg, 2005.

[6] W. Jäger, and A. Mikelić, Couette flows over a rough boundary and drag reduction, in Communications in Mathematical Physics, Springer, Vol. 232, p.429$455,2003$. 